\title{
High PeRformanCES SignAl GENERATOR IMPLEMENTED ON Two Axes Hydraulic Pulsator
}

\author{
Svetlana Despotovic ${ }^{1}$, Zeljko Despotovic ${ }^{1}$, Member IEEE, Srdjan Sudarevic ${ }^{1}$
}

\begin{abstract}
In this paper is presented a specific realization of 2-axe hydraulic vibration exciter, i.e. hydraulic pulsator (vibrator), with the driving system based on hydraulic servo drive and high performances signal generator. This mechanical system presents a part of testing laboratory for vibration influence on a human body researching, with ride comfort point of view. Signal generator is based on Direct Digital Synthesis method (DDS), with possibility of choosing different excitation types (sine wave, triangular wave or stochastic signal) and frequency and amplitude fine tuning. Different signal generation, with frequency range $(0.1-31.5) \mathrm{Hz}$ and amplitude range (0-50) $\mathrm{mm}$, is detailed presented. Experimental results of hydraulic platform displacement (oscilloscopic waveforms and spectrum in frequency domain) over both axes and real mass load for different excitation types from signal generator are presented too.
\end{abstract}

Index Terms: hydraulic pulsator, direct digital synthesis (DDS), signal generator, stochastic signal, PI servo regulator.

\section{INTRODUCTION}

With ride comfort point of view, cognition of vibration transmission across the human body and their influence on psycho-physiological abilities are very important for automotive design. That influence is nowadays researched on real and laboratory conditions. More attention is given on laboratory research, because stability of micro-environment parameters (noise, thermal load etc.) and repeatability are assured [1].

There are two aspects for vibration influences on human body research [2] [3]:

- Aspect of health (fatigue, ride comfort, appearance of professional diseases etc.)

- $\quad$ Aspect of mechanical vibration transmission across the human body (biodynamic).

In modern literature influence of vertical vibration (harmonic at first and after that random) is mostly observed. The frequency range from $1 \mathrm{~Hz}$ to $30 \mathrm{~Hz}$ is commonly of interest.

It is indicated that the man is so sensitive on frequencies less than $1 \mathrm{~Hz}$; like $0.1 \mathrm{~Hz}$, even $0.05 \mathrm{~Hz}$, which is detailed represented in [4],[5],[6]. Mechanical devices, named vibratory exciters, pulsators or vibrators, are utilized in those inquiries. Most frequently, pulsators work on hydraulic principle, due to significant mass load. They are realized like hydraulic platform with excitation opportunity over two independent directions (two axes) or one line direction (one axis). Uncommonly, simultaneously excitation over two axes, i.e. compound movement is required. Driving system for pulsators should be designed to ensure continual amplitude tuning and vibration frequency over both or a single direction. Possibility for different waveforms generation (like sine, triangle or square wave) at a given frequency, or stochastic signal generation [7],[8].

In this paper is presented a specific realization of hydraulic pulsator and its driving system, applied on aforesaid laboratory inspection. The main parts of driving circuit are analog hydraulic servo controlled amplifier and digital signal generator. Output from signal generator is converted, due to digital to analog converter, in analog form which is led on referent input of analog servo PI controller. Signal generator, with possibility of choosing different excitation types and frequency and amplitude output signal tuning is based on Direct Digital Synthesis Method (DDS).

\section{HYDRAULIC PULSATOR CONTROL SYSTEM}

The principal scheme of hydraulic pulsator and its control system is shown in Fig.1. There are several units in this configuration: electrical energy supply, hydraulic part, PI servo controllers, digital control module and system for measurement.

The energizing of electrical motors M1, M2 and appropriate hydraulics pumps $\mathrm{P} 1$ and $\mathrm{P} 2$ is often realized from three-phase AC supply. Feeding of hydraulic installation and appropriate hydraulics pumps P1 and P2 is achieved from oil tank. Hydraulics pumps have constant pressure. In the oil tank are temperature and oil level sensors, which function is to protect hydraulic aggregate. In the direct pipes and back pipe pressure is measured and visual representation is provided.

Load platform (LP) moving is obtained over two perpendicular axes $\mathrm{X}$ and $\mathrm{Z}$ by hydraulic pistons (HP1, HP2). Either hydraulic pistons has corresponding valve manifold (VM1, VM2) and servo valve (SV1, SV2). With displacement probes (DP1, DP2) is accomplished displacement measurement over $\mathrm{X}$ and $\mathrm{Z}$ axe, respectively. Via displacement

\footnotetext{
1 Authors are with Mihailo Pupin Institute, Volgina 15,

11060 Belgrade,Serbia, e-mail:svetlana.despotovic@automatika.imp.bg.ac.yu
} 


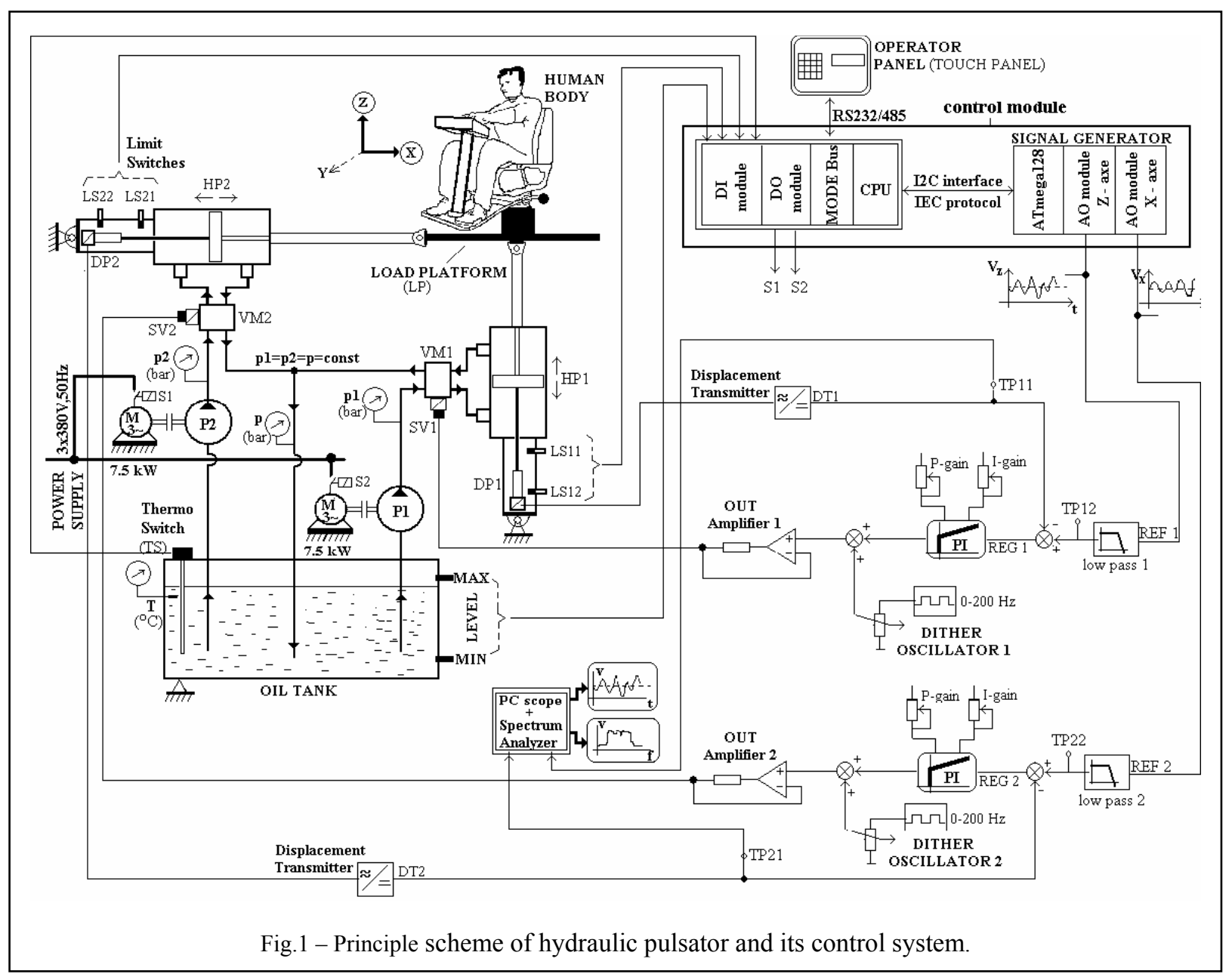

transmitters (DT1, DT2) measured signals from displacement probes (DP1,DP2) are led on servo controller's inputs and test points (TP1, TP2). Detection of end position of hydraulic pistons is reached by limit switches (LS11, LS12 for Z axe and LS21, LS22 for X axe).

The servo control system consists of PI regulators $\mathrm{R} 1$ and R2. The regulators have possibility of P-gain and I-gain tuning. The output signals from R1 and R2 are lead on servo valves SV1, SV2 respectively, over voltage amplifiers. The adjustable Dither oscillators are here for dynamic system feature improvement. Their tuning range is $(0-200) \mathrm{Hz}$. Reference values REF1 and REF2 are lead on servo controller's inputs via low pass filter 1 and low pass filter 2. Reference value generation is possible owing to signal generator which is within digital control module.

Module for digital control has two parts: digital inputs (DI) for discrete signal acquisition and digital outputs (DO) for motor M1 and M2 activation.

Parameters are displayed and can be altered over touch panel. Communication between CPU unit and touch panel is over serial connection RS232/485 and MODEBUS protocol. The customer can select one among three operation mode (NO SWEEP, SWEEP, STOCHASTIC), excitation type (sine wave or triangular wave) and axe of working (horizontal or vertical) over the touch panel. Moreover, there are many parameters on touch panel that could be adjustable (all of them over two channels) - minimal frequency $\left(f_{\min }\right)$, maximal frequency $(f \max )$, total time of working $\left(t_{\text {total }}\right)$, step in the case of SWEEP mode of operation (step).

The signal generator is based on micro processor ATMEL Mega 128 and two 12-bits MAX5322 digital to analog converters, for analog output (AO) signals reaching (one for $\mathrm{Z}$, the other one for $\mathrm{X}$ axe). Micro processor has four timers: two of them are 8-bits (for total time of working control), the other two are 16bits (their interrupts are directly depended on desirable resolution frequency). ATMEL Mega 128 communicates with CPU over I2C interface with IEC protocol.

Principle of signal generation is reposed on Direct Digital Synthesis (DDS) method. This method will be explained in the next section.

\section{DIRECT DIGITAL SYNTHESIS METHOD}

Direct digital synthesis (DDS) is a method of producing an analog waveform, usually a sine wave (but triangular and square waves are inherent) at a given frequency [9]. The frequency depends on two variables: the reference clock frequency and the binary number programmed into the frequency 
register (tuning word) [10]. The binary number in the frequency register provides the main input to the phase accumulator. If a sine lookup table is used, the phase accumulator computes a phase (angle) address for the lookup table, which outputs the digital value of amplitude - corresponding to the sine of that phase angle - to the DAC. The DAC, in turn, converts that number to a corresponding value of analog voltage or current (voltage in this case), like in Fig 2.

To generate a fixed frequency sine wave, a constant value (the phase increment, which is determined by the binary number) is added to the phase accumulator with each clock cycle.

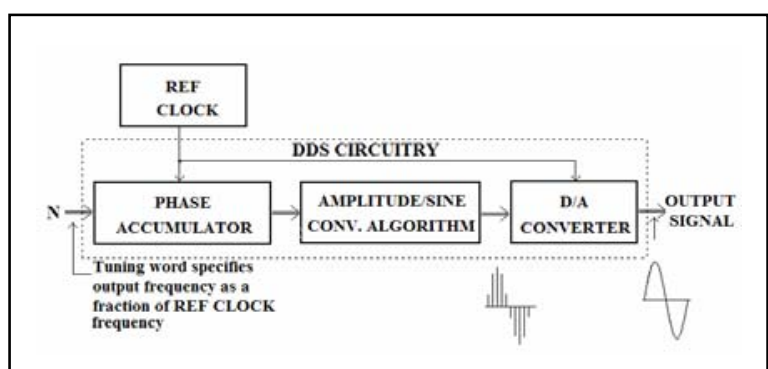

Fig. 2 - Components of a direct digital synthesizer.

If the phase increment is large, the phase accumulator will step quickly through the sine lookup table and thus generate a high frequency sine wave. If the phase increment is small, the phase accumulator will take much more steps, accordingly generating a slower waveform.

\section{IV.HYDRAULIC PULSATOR OPERATION MODES}

There are three modes of hydraulic pulsator operation:

NO SWEEP - In this mode of operation, sine or triangle wave will be generated over the whole time range, $t_{\text {total }}$, with amplitude $A$ and with the same operating frequency.

SWEEP - A sweep function is realized over the chosen frequency range from $f_{\min }$ to $f$ max, or vice versa with the same amplitude $(A)$, with selected step (step) and during the total time of working ( $\left.t_{\text {total }}\right)$. The minimal step of frequency changing is $0.1 \mathrm{~Hz}$ (that is withal the maximal resolution), so after some time which is software determined, frequency will be changed from $f_{\min }$ to $f_{\min }+$ step, apropos from $f_{\max }$ to $f_{\max }-$ step. The last generated frequency will be $f_{\max }$, apropos $f_{\min }$, respectively.

STOCHASTIC - Parameters for this working mode are: amplitude, minimal and maximal frequency and the total time of work. The only excitation type is a sine wave. The stochastic signal is produced from the sum of sine functions at the same time interval, which depends on the frequency range and can vary from $2.5 \mathrm{~ms}$ to $10 \mathrm{~ms}$, as will be explained later in the next section. All sine functions have the same amplitude, different frequencies (from $f_{\text {min }}$ to $\left.f_{\max }\right)$ and the random initial phases. Because of that the resulting signal will be random, i.e. stochastic.

\section{DIFFERENT SIGNAL GENERATION}

At the beginning of this section, in Table 1, is presented required ranges for frequency, amplitude and time of working.

Table 1. Required ranges for frequency, amplitude and total time of working.

\begin{tabular}{|l|l|}
\hline Frequency & $(0.1-31.5) \mathrm{Hz}$ \\
\hline Amplitude & $(0-50) \mathrm{mm}$ \\
\hline Total time of working & $(0-999) \mathrm{min}$ \\
\hline
\end{tabular}

With a view to generate different signals for platform driving, there are two lookup tables, one for sine wave and the other one for triangle wave generation.

In the case of SWEEP or NO SWEEP operation mode, desirable resolution is $0.1 \mathrm{~Hz}$, which is contained a whole number of times, like all other frequencies from range given in Table 1 , in $0.01 \mathrm{~Hz}$ frequency. So, we can proclaim that $0.01 \mathrm{~Hz}$ frequency is the new smallest frequency, i.e. resolution frequency, which determines how finely we decompose the signal. The period of resolution frequency, which is $100 \mathrm{~s}$, directly affect on sampling time, i.e. occurring interrupts. In these interrupts, the desirable samples are sent to digital to analog conversion input which converts that number to a corresponding value of analog voltage.

One complete sine cycle is stored in the 40000 location sine lookup table (contains amplitude value for each of 40000 samples), which is used for SWEEP/NO SWEEP sine signal and stochastic signal generation (while the other lookup table is used only for triangle wave generation in SWEEP/NO SWEEP working mode). If the phase accumulator output increments by one on each clock, then all the stored sine-wave values will be read out consecutively by the time of $100 \mathrm{~s}$. That means that each interrupt will be occurred after $2.5 \mathrm{~ms}$. On each clock, the output phase will advance by $360 / 40000=0.009$ degrees.

In the case of NO SWEEP/SWEEP working mode with sine excitation, for generating $0.1 \mathrm{~Hz}$ sine signal, every tenth point from sine lookup table will be read.

One complete triangle cycle is stored in the 4000 location lookup table. And if the phase accumulator output increments by one on each clock (that corresponds to $0.1 \mathrm{~Hz}$ frequency), then all stored triangle-wave values will be read out consecutively. On each clock, the output phase will advance by $360 / 4000=0.09$ degrees. That means that each interrupt will be occurred after $2.5 \mathrm{~ms}$.

It is already mentioned in the previous section that 
the stochastic signal can be represented by a sum of sinusoid functions with different frequency, different initial phase and the same amplitude. Those sinusoid functions are added at the same time intervals, which varied from $2.5 \mathrm{~ms}$ to $10 \mathrm{~ms}$ due to satisfactory number of sinusoid functions per frequency interval $\left(\Delta f=f_{\max }-f_{\min }\right)$.

It is advisable existence of a great number of sine functions per frequency interval. Nevertheless, attainable number of sine function is restricted by two factors: processor's speed (which directly affect on the number of sine waves, because it restricts number of arithmetic operations in timers interrupt that can be achieved without signal distortion) and the fact that $10 \mathrm{~ms}$ is the maximal time for timer interrupt due to motor features $(10 \mathrm{~ms}$ interrupt corresponds to $100 \mathrm{~Hz}$ frequency, which is chattering frequency for this system). That frequency influenced on the servo valves and hydraulic cylinder, so the flutter of hydraulic platform is occurred and the signal is distorted. Due to, the maximal $10 \mathrm{~ms}$ interrupt provides maximal resolution which is $0.0025 \mathrm{~Hz}$ ). The resolution is better if the interrupt occurs after greater time. With this hardware configuration and due to motors, the best resolution is $0.0025 \mathrm{~Hz}$.

Every customer can select any frequency range between boundaries given in Table 1. Some required ranges, important for pulsators working are in the left column of Table 2, while obtained sine number is in the right column (explanation is given later in this section).

Table 2. Some required frequency ranges.

\begin{tabular}{|l|l|}
\hline $\begin{array}{c}\text { Frequency range } \\
\text { [Hz] }\end{array}$ & \multicolumn{1}{|c|}{$\begin{array}{c}\text { Number of } \\
\text { sine }\end{array}$} \\
\hline$(0.10-0.125)$ & 11 \\
\hline$(0.125-0.16)$ & 10 \\
\hline$(0.16-0.20)$ & 10 \\
\hline$(0.20-0.25)$ & 12 \\
\hline$(0.25-0.315)$ & 14 \\
\hline$(0.315-0.40)$ & 20 \\
\hline$(0.40-0.50)$ & 11 \\
\hline$(0.50-0.63)$ & 14 \\
\hline$(0.63-0.80)$ & 18 \\
\hline$(0.10-31.5)$ & 31 \\
\hline
\end{tabular}

The maximal sine number that can be added in timer's interrupt is 50 per channel, but it is decided that a number of 21 sine is quite enough for all frequency ranges where it is possible, i.e. where is $\Delta f>0.19$ (with maximal resolution of $0.01 \mathrm{~Hz}$ ). Since there are many ranges with $\Delta f \leq 0.19$, the resolution should be better than $0.01 \mathrm{~Hz}$. There are several ranges with $0.05 \mathrm{~Hz}$ resolution, even with $0.0025 \mathrm{~Hz}$ resolution. These ranges are represented in Table 3. Now is clear why the right column in Table 2 is just like that.

Table 3. Number of sine functions per frequency range and with determined resolution.

\begin{tabular}{|l|l|l|}
\hline $\begin{array}{c}\text { Frequency } \\
\text { range } \Delta f[\mathbf{H z}]\end{array}$ & Precision [Hz] & $\begin{array}{c}\text { Number of sine } \\
\text { functions }\end{array}$ \\
\hline$\Delta f>0.19$ & $\max 0.01$ & 21 \\
\hline $0.09<\Delta f<0.2$ & 0.01 & $\Delta f * 100+1$ \\
\hline $0.03<\Delta f<0.1$ & 0.005 & $2 * \Delta f * 100+2$ \\
\hline$\Delta f<0.04$ & 0.0025 & $4 * \Delta f * 100+3$ \\
\hline
\end{tabular}

\section{RANDOM INITIAL PHASE GENERATION AND AMPLITUDE SCALLING}

In respect of random initial phase determination, the algorithm [11] is implemented. Let's assume that a counter, named counter[0], represents the initial phase for the first sine function, i.e. the sine function with the smaller frequency in desired range. That initial phase, initial_phase0, is obtained from a micro controllers' timer, which stopped at the moment of initializing the whole initial conditions for pulsator's work. The flow chart for random initial phase generation is given in Fig 3.

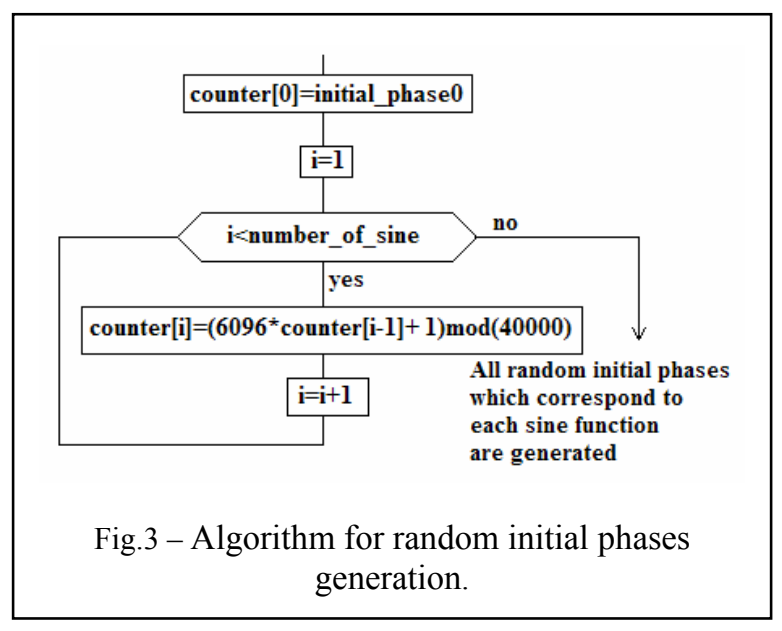

The digital to analog converter has 12-bits resolution and provides (0-10) $\mathrm{V}$ on its output. All values of interest should be mapped on a number range of (0-4094). The amplitude resolution in every mode of operation is $0.1 \mathrm{~mm}$ and the number of 4.094 corresponds to it. For stochastic signal, which consists of $n$ sinusoid functions, amplitude of every sinusoid is decreased by $n$ times.

\section{EXPERIMENTAL RESULTS}

Previously depicted ways of driving signal generation and experimental verified on two axes hydraulic platform. In this section is presented measurement values of hydraulic platform movements, as well as frequency features at stationary excitation (sine and triangle wave in 
SWEEP/NO SWEEP mode of operation).

The energizing of electrical motors and appropriate hydraulic pumps is realized from the mains supply $3 \times 380 \mathrm{~V}, 50 \mathrm{~Hz}$. Power rating of motors is $7.5 \mathrm{~kW}$ apart.

Hereafter are given time response and frequency analysis over stationary and stochastic (random) excitation. Experimental results are obtained upon some conditions, presented in Table 4

Table 4. Coditions for experimental results obtaining.

\begin{tabular}{|l|l|}
\hline Pressure in hydraulic installation & $130 \mathrm{bar}$ \\
\hline Platform mass load & $200 \mathrm{~kg}$ \\
\hline Displacement probe (vertical axe) & $11.7 \mathrm{~mm} / \mathrm{V}$ \\
\hline Displacement probe (horizontal axe) & $11.0 \mathrm{~mm} / \mathrm{V}$ \\
\hline
\end{tabular}

In the Fig. 4 - Fig.6 are represented oscilloscopic records of set and realized values for sine wave oscillation over two axes, between minimal and maximal frequency. $\mathrm{CH} 1$ and $\mathrm{CH} 2$ are labels for referenced and realized displacement values. In Fig.4 is exposed record for vertical axe excitation. It is obtained $40 \mathrm{~mm}$ amplitude under $0.1 \mathrm{~Hz}$ frequency. It is obvious that realized signal precisely follows the setting value over amplitude and frequency.

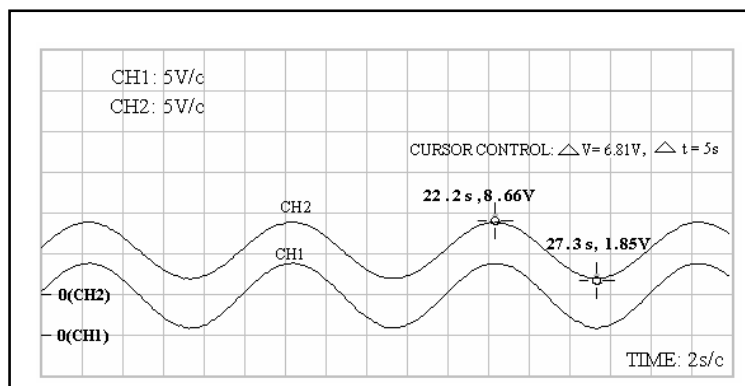

Fig. 4 - Stationary sine excitation over vertical axe. Amplitude is $40 \mathrm{~mm}$, frequency $0.1 \mathrm{~Hz}$.

In Fig. 5 are presented waveforms for vertical axe excitation. It is obtained $1 \mathrm{~mm}$ amplitude under frequency of $30 \mathrm{~Hz}$. It is obvious that realized signal precisely follow the setting value over amplitude, but there is some deviation of frequency. The reason for that is in fact that servo regulator hasn't got adaptive parameters tuning (but compensation of this phase bias wasn't in the list of requirements, although it could be exceeded by adaptive control).

In Fig.6 are waveforms of sine excitation over horizontal axe. It is obtained $40 \mathrm{~mm}$ under $0.1 \mathrm{~Hz}$ frequency. Realized signal precisely follows the setting values.

In Fig. 7 and Fig. 8 are given records of stationary triangle wave excitation over vertical axe. It is attained $40 \mathrm{~mm}$ amplitude for $0.1 \mathrm{~Hz}$ (Fig.7), apropos $3 \mathrm{~mm}$ for $10 \mathrm{~Hz}$ frequency (like in Fig. 8).

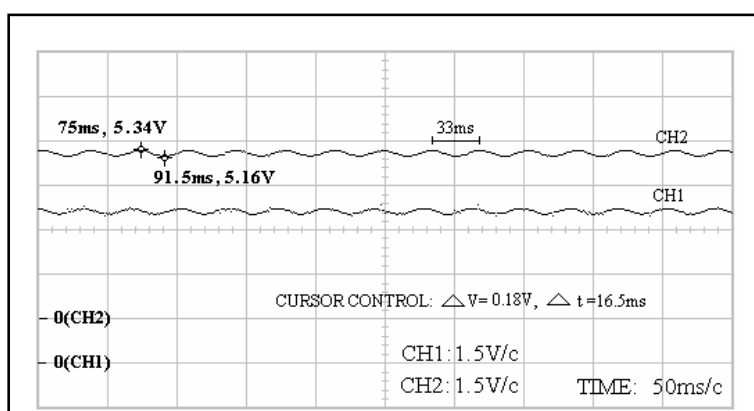

Fig.5 - Stationary sine excitation over vertical axe. Amplitude is $1 \mathrm{~mm}$, frequency $30 \mathrm{~Hz}$.

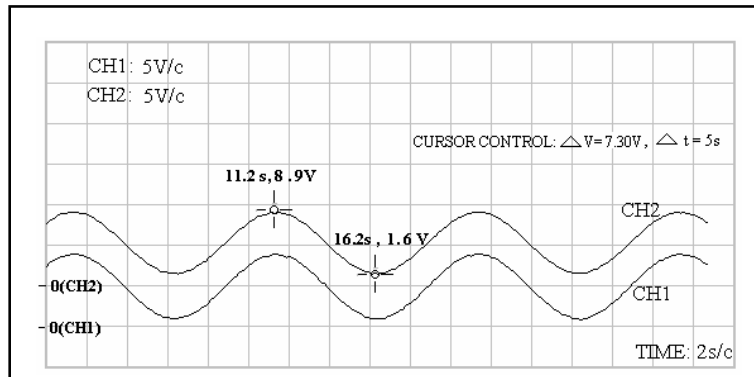

Fig.6 - Stationary sine excitation over horizontal axe. Amplitude is $40 \mathrm{~mm}$, frequency $0.1 \mathrm{~Hz}$.

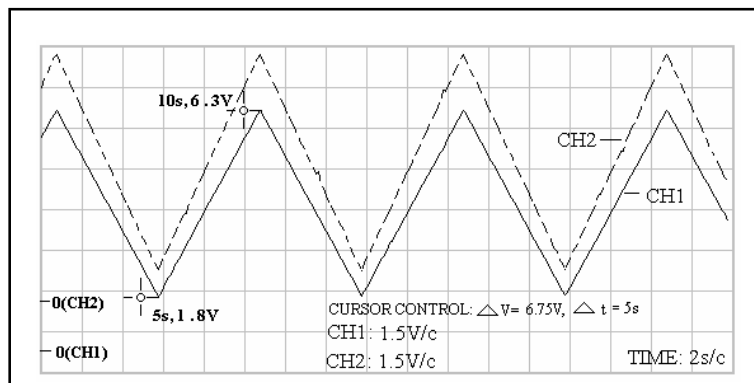

Fig.7 - Stationary triangle excitation over vertical axe. Amplitude is $40 \mathrm{~mm}$, frequency $0.1 \mathrm{~Hz}$.

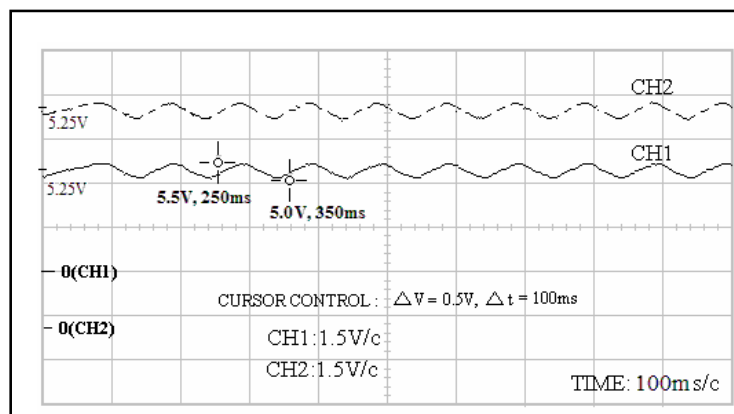

Fig.8 - Stationary triangle excitation over vertical axe. Amplitude is $3 \mathrm{~mm}$, frequency $10 \mathrm{~Hz}$.

In both cases in figures Fig. 7 and Fig. 8 is noticed precisely following over setting values for amplitude 
and phase.

In Fig.9 is shown system response in frequency (upper) and time domain (below), on stochastic excitation with $40 \mathrm{~mm}$ amplitude and frequency range (0.1-0.125) Hz. Attained stochastic signal corresponds to initial excitation in amplitude, as well as frequency range.

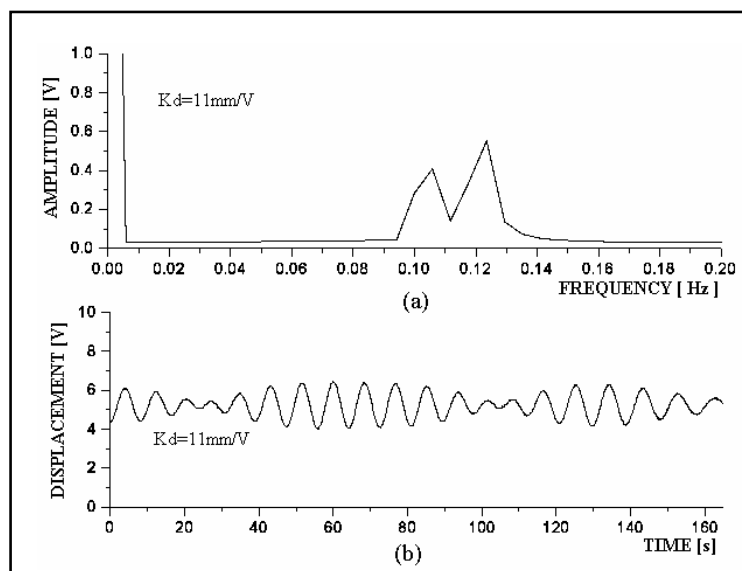

Fig.9 - Stochastic excitation over horizontal axe. Amplitude is $40 \mathrm{~mm}$, frequency range $(0.1-0.125) \mathrm{Hz}$. Sum of 11 sine waves is obtained.

Similar conclusions can be accomplished for some other stochastic excitations: in Fig.10 (amplitude is $40 \mathrm{~mm}$, frequency range $(3.15-4) \mathrm{Hz}$ ), in Fig.11 (amplitude 3mm, frequency range $(12.5-16) \mathrm{Hz}$ ) and Fig.12 (amplitude 1mm, frequency range (20-25) Hz).

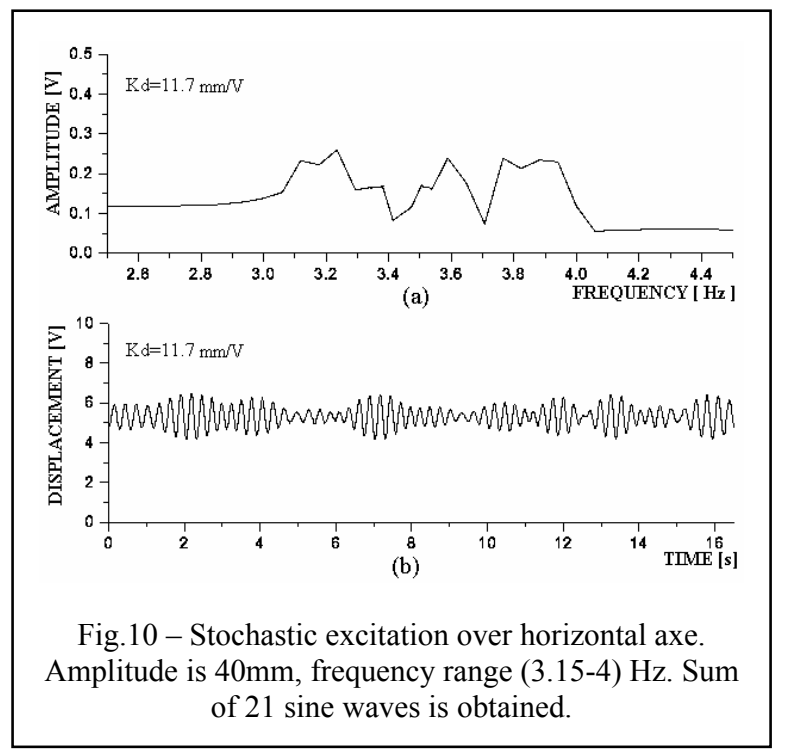

In Fig.13 is presented amplitude-frequency characteristic of the whole system of hydraulic pulsator, for amplitude operating range (0-50) $\mathrm{mm}$ and frequency operating range $(0.1-40) \mathrm{Hz}$.
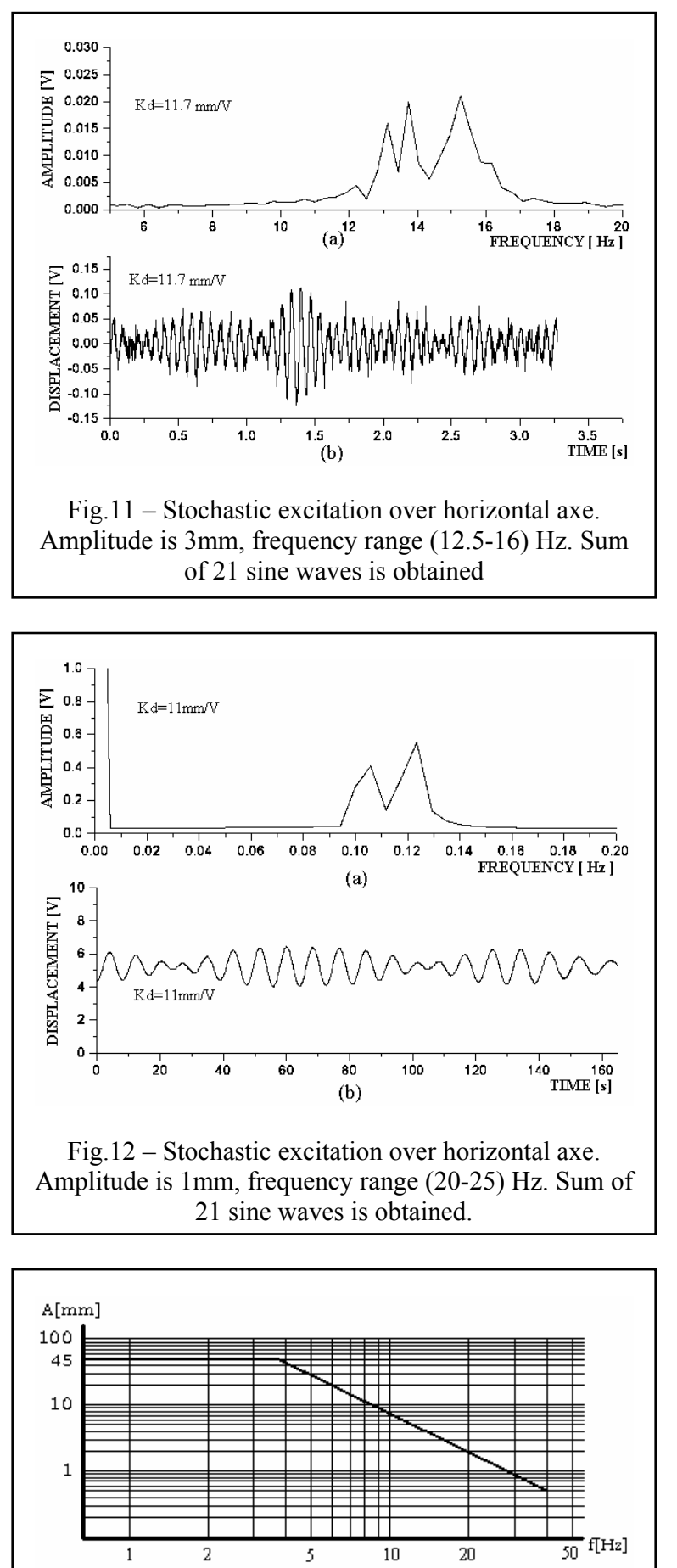

Fig.13 - Amplitude-frequency diagram of hydraulic pulsator in operating range.

\section{CONCLUSION}

In this paper is exposed a specific realization of hydraulic pulsator and its driving system for vibration in two perpendicular directions. Desirable signals are generated from signal generator. Amplitude values near $40 \mathrm{~mm}$ are possible in frequency range (0.1-4) 
$\mathrm{Hz}$, while smaller amplitude values corresponds to greater frequency ranges, as shown on amplitudefrequency ranges, as shown on amplitude-frequency characteristics, in Fig.13. Servo amplifier obtains fine following of initial (desirable) value.

A DDC method, which is used for different waveforms generation is software implemented.

Thereafter, some important results are represented, which are relates on stationary (harmonic) excitation and stochastic excitation with different frequency ranges.

The overall presented system is installed in the department for motor vehicles and motors at the faculty of mechanical engineering, University of Kragujevac.

\section{REFERENCES $^{2}$}

[1]. Griffin M., Handbook of Hyman Vibration, San Diego: Academic Press, 1990

[2]. Dupuis H., Zerlett G. Beanspruchung des Mencshen durch Mechanishe Schwingungen, Westkreuz, BerlinBon, 1984

[3]. Simic.D., The Influence of Horizontal and Transversal Oscillations on the Man, UK, Informal Group on Human Response to Vibration, Silsoe, 1978.

[4]. Demic.M., "A Contribution to Definition of Tolerable Level of Random Vertical Vibration Loads of Bus Underbody from the Aspect of Physiology", 15 Conference of bus and coach experts, Budapest, Hungary, 1984

[5]. Demic.M., “ Assessment of Random Vertical Vibration on Human Body Fatigue Using Psychological Approach“", IMeCHE, C-153/84, London, 1984

[6]. Demic.M., Lukić.J, Milic.Z. "Some Aspects of the Investigation of Random Vibration Influence on Ride Comfort", Journal of Sound and Vibration, Vol.253, Issue(1),2002,pp.109-129.

[7]. Demic.M., Lukic.J., Investigation of the transmission of fore and aft vibration through the human body, Applied Ergonomics, In Press, Corrected Proof, Available online, 16 July 2008.

[8]. Demic S.M., Lukic, K.J, "Human Body Under TwoDirectional Random vibration", Journal of Low Frequency Noise, Vibration and Active Control, Vol. 27, No. 3. (September 2008), pp.185-201.

[9]. "All about Direct Digital Synthesis" [Online]

${ }^{2}$ Svetlana B. Despotovic, born in 1973, Serbia. Received Dipl. Ing. degree in Faculty of Electrical Engineering at Belgrade University. She has been working at Institute "Mihajlo Pupin" since 2000, as research and development engineer on department for Industrial Automation.

Zeljko V. Despotovic, born in 1964, Serbia. Received Dipl. Ing., M.Sc. and Ph.D. degrees in Faculty of Electrical Engineering at Belgrade University. $\mathrm{He}$ has been with Institute "Mihajlo Pupin" since 1991. IEEE member since 2004. His research interests include the fields of power electronics, mechatronics, control of power converters and vibration control.

Srdjan R. Sudarevic, born in 1968, Serbia. Graduate on Military Faculty at Belgrade University, department for electronics. He is employee of Institute "Mihajlo Pupin" in Belgrade, on department for Industrial Automation.
Available at: www.analog.com/analogdialogue

[10]. "Function generators offer more than sine waves; with many standard waveforms and types of modulation, a modern function generator almost runs tests by itself", [Online], Available at: http://findarticles.com/p/articles/mi_hb4797/is_/ai_n29 022875

[11]. "Random Number Generation" [Online], Available at: http://linux.duke.edu/ mstenner/free-docs/gsl-ref1.1/gsl-ref_17.html 